\title{
TAB3 promotes human esophageal squamous cell carcinoma proliferation and invasion via the $\mathrm{NF}-\kappa \mathrm{B}$ pathway
}

\author{
JIYI ZHAO ${ }^{1 *}$, LING GAI $^{2 *}$, YI GAO $^{1 *}$, WENKAI XIA $^{3}$, DONG SHEN $^{4}$, QINGFENG LIN $^{4}$, \\ WEIDONG MAO ${ }^{4}$, FANGJUN WANG ${ }^{1}$, PENGFEI LIU ${ }^{1}$ and JIE CHEN ${ }^{4}$ \\ ${ }^{1}$ Department of Gastroenterology, The Jiangyin Clinical College of Xuzhou Medical University, Wuxi, Jiangsu 214400; \\ ${ }^{2}$ Department of Oncology, The Affiliated Hospital of Nantong University, Nantong, Jiangsu 226001; \\ Departments of ${ }^{3}$ Nephrology and ${ }^{4}$ Oncology, The Jiangyin Clinical College of \\ Xuzhou Medical University, Wuxi, Jiangsu 214400, P.R. China
}

Received February 4, 2018; Accepted September 3, 2018

DOI: $10.3892 / o r .2018 .6686$

\begin{abstract}
Esophageal squamous cell carcinoma (ESCC) has become one of the most common causes of cancer-associated mortality worldwide. Transforming growth factor-activated kinase (TAK1)-binding protein 3 (TAB3) is essential for activation of the NF (NF)- $\kappa$ B pathway in response to TAK1 activation. The $\mathrm{NF}-\kappa \mathrm{B}$ pathway serves important roles in tumor cell proliferation and migration; however, the clinical relevance of TAB3 and its biological function in ESCC progression remain elusive. The present study investigated the expression and function of TAB3 in ESCC tissues, and its association with the clinical prognosis of patients. The results demonstrated that TAB3 expression was significantly increased in human ESCC cell lines and tissue samples, and the expression of TAB3 was associated with ESCC lymph node metastasis, $\mathrm{T}$ stage, pathological grade and $\mathrm{Ki}-67$ expression in 80 ESCC samples, as determined by immunohistochemistry. Patients with ESCC and high TAB3 expression exhibited worse overall survival. Furthermore, knockdown of TAB3 by small interfering RNA inhibited the proliferation of ESCC cells, and reduced the migration and invasion of ESCC cells. In addition, knockdown of TAB3 decreased the expression of the NF- $\kappa \mathrm{B}$ pathway in TE-1 cells. Taken together, these results demonstrated that TAB3 may be a promising therapeutic target for the treatment of ESCC.
\end{abstract}

Correspondence to: Professor Pengfei Liu, Department of Gastroenterology, The Jiangyin Clinical College of Xuzhou Medical University, 163 Shoushan Road, Wuxi, Jiangsu 214400, P.R. China E-mail: pengfeimd@163.com

Ms. Jie Chen, Department of Oncology, The Jiangyin Clinical College of Xuzhou Medical University, 163 Shoushan Road, Wuxi, Jiangsu 214400, P.R. China

E-mail: chenjie0819@126.com

${ }^{*}$ Contributed equally

Key words: esophageal squamous cell carcinoma, transforming growth factor-activated kinase-binding protein 3 , nuclear factor- $\kappa \mathrm{B}$

\section{Introduction}

Human esophageal squamous cell carcinoma (ESCC) has a very high incidence, and has become one of the most common causes of cancer-associated mortality worldwide (1,2). Surgical resection and radiotherapy are the predominant therapeutic interventions used to treat patients with ESCC, whereas neoadjuvant chemotherapy and photodynamic therapy have been considered promising alternative strategies (3). Despite advances in clinical and experimental oncology methods, patients with ESCC often have a poor prognosis (2-5). ESCC is associated with a poor outcome due to its malignant biological characteristics, including histopathological grade, and early metastasis and invasion (6,7). The etiology of ESCC is a complex process, and the exact molecular mechanism remains unclear. Therefore, the identification of pathogenetic mechanisms that occur during ESCC progression and metastasis is a priority in ESCC research.

Human transforming growth factor-activated kinase (TAK1)-binding protein 3 (TAB3) is essential for TAK1 activation $(8,9)$. TAK1 is an activator of nuclear factor $(\mathrm{NF})-\kappa \mathrm{B}$, which serves critical roles in various cellular processes that contribute to embryonic development, immunity, cell survival, carcinogenesis, chemoresistance and others (10). These findings indicate that the TAB3-TAK1-NF- $\kappa$ B pathway may be central to numerous cellular processes. In addition, it has been reported that TAB3 is overexpressed in several types of cancer, including breast, ovarian and lung cancer, and that it is associated with tumor development and invasion (11-13). However, the role of TAB3 in the development of ESCC remains to be elucidated. The present study aimed to explore the expression and function of TAB3 in ESCC cells. The results indicated that TAB3 may enhance the proliferation and invasion of ESCC cells; therefore, it may be of value with regards experimental therapeutic strategies for the treatment of ESCC.

\section{Materials and methods}

Cell lines and cell culture. Human esophageal epithelial cells (HEECs), and the TE-1, TE-10 and Eca-109 cell lines were purchased from the Shanghai Institute of Cell Biology 
(Shanghai, China). The cells were maintained in Dulbecco's modified Eagle's medium (DMEM; Gibco; Thermo Fisher Scientific, Inc., Waltham, MA, USA) supplemented with $10 \%$ fetal bovine serum (FBS; Hangzhou Sijiqing Biological Engineering Materials Co., Ltd., Hangzhou, China) at $37^{\circ} \mathrm{C}$ in a humidified atmosphere containing $5 \% \mathrm{CO}_{2}$.

Antibodies. The antibodies used in the present study were as follows: Anti-TAB3 (cat. no. sc-166538, 1:500), anti-GAPDH (cat. no. sc-166574, 1:1,000), anti-Ki-67 (cat. no. sc-23900, 1:100), anti-cyclin D1 (cat. no. sc-450, 1:300), anti-proliferating cell nuclear antigen (PCNA; cat. no. sc-25280, 1:1,000), anti-TAK1 (cat. no. sc-7967, 1:300) and anti-p65 (cat. no. sc-71675, 1:100), all of which were purchased from Santa Cruz Biotechnology, Inc. (Dallas, TX, USA).

Tissue samples. Paired ESCC and adjacent non-cancerous tissues were obtained from 80 patients who underwent surgery between June 2010 and September 2012 at the Department of Pathology of the Affiliated Hospital of Nantong University (Nantong, China). No patient had received chemotherapy or radiotherapy prior to surgery. Patients with a family history of gastric/colorectal cancer were excluded. All patients provided written informed consent for their tissue samples to be used for scientific research. The present study was approved by the Affiliated Hospital of Nantong University Ethics Committee. The histological features of the specimens were evaluated by a senior pathologist, according to the World Health Organization classification criteria (14). All patients were followed up for 4.2-64.9 months. Patient follow-up was terminated on November 30, 2016. The main clinical and pathological characteristics of the patients are presented in Table I.

Immunohistochemistry (IHC). For histological examination, all surgically excised tissues were fixed with $10 \%$ formalin at $4^{\circ} \mathrm{C}$ for $24 \mathrm{~h}$ and embedded in paraffin; subsequently, $4-\mu \mathrm{m}$ specimen sections were prepared on glass slides. The sections were deparaffinized in xylene and rehydrated with graded alcohol washes, and antigen retrieval was performed by heating the samples to $121^{\circ} \mathrm{C}$ for $3 \mathrm{~min}$ in $10 \mathrm{mM}$ citrate buffer ( $\mathrm{pH} \mathrm{6.0)}$. Hydrogen peroxide (0.3\%) was applied to block endogenous peroxide activity for $20 \mathrm{~min}$ after cooling, in order to block any nonspecific reactions. After rinsing in PBS ( $\mathrm{pH} 7.2)$, the sections were incubated with rabbit anti-human TAB3 (1:100) for $2 \mathrm{~h}$ at room temperature. All slides were processed using the peroxidase-antiperoxidase method (EnVision + Dual Link system-horseradish peroxidase; Dako; Agilent Technologies, Inc., Santa Clara, CA, USA), according to the manufacturer's protocol. After washing with PBS, the peroxidase reaction was visualized by incubating the sections with $\mathrm{DAB}(0.1 \%$ phosphate buffer solution, $0.02 \%$ diaminobenzidine tetrahydrochloride and $3 \% \mathrm{H}_{2} \mathrm{O}_{2}$ ) at room temperature for $5 \mathrm{~min}$. After being rinsed in water, the sections were counterstained with hematoxylin $(0.5 \%)$ at room temperature for $1 \mathrm{~min}$. Finally, the sections were dehydrated with graded alcohol washes leicand cover slips were added to the slides, which were observed under a microscope (Leica DFC 300 FX; Leica Microsystems, Inc., Buffalo Grove, IL, USA).

Evaluation of the results of immunohistochemical staining. Three observers who were blind to the clinical and follow-up data evaluated the staining results independently using a multihead microscope. Sections were co-observed to reach a consensus when the evaluations were divergent. For assessment of TAB3, >1,000 cells from five high-power fields in each specimen were randomly selected, and the staining was examined to determine the mean percentage. The IHC staining was scored according to the following method: Intensity of TAB3 staining was scored as 1 (negatively or poorly stained), 2 (moderately stained) or 3 (strongly stained). Percentage scores were assigned as $1(0-49 \%), 2(50-74 \%)$ or $3(75-100 \%)$ for univariate analyses. After multiplying the two scores, the specimens were divided into two groups according to the scores (average $=4.5$ ): Score $\geq 4.5$ was defined as the high expression group and score $<4.5$ was defined as the low expression group.

For Ki-67 detection, tissues were deparaffinized in toluene, rehydrated in a graded series of ethanol solutions and processed for immunohistochemistry. Briefly, after incubation with $0.3 \%$ hydrogen peroxide for $30 \mathrm{~min}$ at room temperature to quench endogenous peroxidase, and heating for $15 \mathrm{~min}$ in $0.1 \mathrm{M}$ citrate buffer ( $\mathrm{pH}$ 6.0) in a microwave oven for antigen retrieval, the sections were blocked with normal serum (Vector Laboratories Inc., Burlingame, CA, USA) corresponding to the origin of the secondary antibodies for $30 \mathrm{~min}$ at room temperature. The slides were incubated overnight at $4^{\circ} \mathrm{C}$ with primary antibodies against Ki-67 (cat. no. sc-23900, 1:100; Santa Cruz Biotechnology, Inc.), After washing with PBS, the slides were incubated with biotinylated secondary antibodies (cat. no. SE131, 1:100; Beijing Solarbio Science \& Technology Co., Ltd., Beijing, China) for $30 \mathrm{~min}$ at room temperature, followed by peroxidase-conjugated avidin-biotin complex for $30 \mathrm{~min}$ at room temperature. Immunostaining was visualized using 3,3'-diaminobenzidine as a chromogen. The slides were then counterstained with hematoxylin. All sample sections were observed under a microscope (Leica DFC 300 FX; Leica Microsystems, Inc.). For determination of the Ki-67 proliferation index, positively stained cells and nuclei were counted on a minimum of six randomly selected fields from representative tumor sections. The proliferative index was calculated as the number of Ki-67-positive cells divided by the total number of cells counted. In half of the samples, the staining was repeated three times to avoid possible technical errors, and similar results were obtained in these samples.

Western blotting. Cells were collected for immunoblotting analysis, washed three times with ice-cold PBS and resuspended in 2X lysis buffer $(50 \mathrm{mM}$ Tris- $\mathrm{HCl}, 120 \mathrm{mM} \mathrm{NaCl}$, $0.5 \%$ Nonidet P-40, $100 \mathrm{mM} \mathrm{NaF}, 200 \mathrm{IM} \mathrm{Na}_{3} \mathrm{VO}_{4}$ and protease inhibitor mixture). Cell lysates were centrifuged at $16,000 \mathrm{x} \mathrm{g}$ for $30 \mathrm{~min}$ at $4^{\circ} \mathrm{C}$, and were then denatured at $100^{\circ} \mathrm{C}$ for $15 \mathrm{~min}$. The total protein concentration was determined using the Bio-Rad protein assay kit (Bio-Rad Laboratories, Inc., Hercules, CA, USA). All protein samples were stored at $-20^{\circ} \mathrm{C}$.

The proteins $(30 \mu \mathrm{g} /$ well) were resolved by $10 \% \mathrm{SDS}-\mathrm{PAGE}$ and were then transferred onto polyvinylidene difluoride membranes. The membranes were blocked with 5\% non-fat milk in TBST (20 mM Tris, $150 \mathrm{mM} \mathrm{NaCl}$ and $0.05 \%$ Tween20) for $2 \mathrm{~h}$ at room temperature, and were then incubated with primary antibodies for $\geq 6 \mathrm{~h}$ at room temperature. After being washed three times, the membranes were incubated with horseradish peroxidase-conjugated secondary human antibodies 
(cat. no. 31430, 1:5,000; Pierce; Thermo Fisher Scientific, Inc.) for $2 \mathrm{~h}$ at room temperature, according to the manufacturer's protocol. The bands were then detected by enhanced chemiluminescence detection systems (Pierce; Thermo Fisher Scientific, Inc.). The band intensity was measured using an ImageJ (version 1.8.0; National Institutes of Health, Bethesda, MD, USA).analysis system (National Institutes of Health).

Transient transfection. The TAB3 small interfering (si)RNA and control siRNA were purchased from Shanghai GeneChem Co., Ltd. (Shanghai, China). The TAB3-specific siRNA target sequence was 5'-GGTTGAAGTCTGAAGTTAA-3' and the control-siRNA sequence was 5'-TTCTCCGAACGTGTCACG T-3'. The human ESCC cell line TE-1 was grown in dishes until the cells reached $80 \%$ confluence, and were then transfected with either control siRNA or TAB3-siRNA (50 nM) using Lipofectamine ${ }^{\circledR} 2000$ (Invitrogen; Thermo Fisher Scientific, Inc.), according to the manufacturer's protocol. The medium was replaced after $24 \mathrm{~h}$ with fresh medium for transfection. The cells in the mock group were untreated. Cells were collected for western blot analysis, wound healing assays, Transwell assays and cell proliferation assays $36 \mathrm{~h}$ post-transfection.

Cell proliferation assays. Cell proliferation was determined using a commercial Cell Counting Kit (CCK)-8 assay (Dojindo Molecular Technologies, Inc., Kumamoto, Japan), according to the manufacturer's protocol. Briefly, cells were plated into a 96-well plate at a density of $2 \times 10^{4}$ cells/well and were allowed to grow overnight. CCK-8 reagent was then added to the wells for $2 \mathrm{~h}$ at $37^{\circ} \mathrm{C}$, and the absorbance was read at a wavelength of $490 \mathrm{~nm}$ using an automated plate reader. The experiments were repeated at least three times.

Cell cycle analysis. Serum starvation and a refeeding process were used to synchronize the cell cycle. Briefly, TE-1 cells were incubated in FBS-free DMEM for $72 \mathrm{~h}$ to synchronize the cells, after which the medium was replaced with complete medium. Subsequently, the cells were rapidly harvested after $48 \mathrm{~h}$, fixed in $70 \%$ ethanol for $\geq 24 \mathrm{~h}$ at $-20^{\circ} \mathrm{C}$, and incubated with $1 \mathrm{mg} / \mathrm{ml}$ RNase A (Invitrogen; Thermo Fisher Scientific, Inc.) for $30 \mathrm{~min}$ at $37^{\circ} \mathrm{C}$. Subsequently, the cells were stained with propidium iodide ( $50 \mu \mathrm{g} / \mathrm{ml}$ ) (BD Biosciences, San Jose, CA, USA) in PBS with $0.5 \%$ Triton X-100, and were analyzed using a BD FACScan flow cytometer (BD Biosciences) with CellQuest acquisition and analysis software (version 1.8.0; BD Biosciences, Franklin Lakes, NJ, USA).

Wound healing assay. Cells were grown in a monolayer in 6 -well plates to nearly $100 \%$ confluence. Subsequently, cells were serum-starved for $12 \mathrm{~h}$ post-transfection with control siRNA or TAB3 siRNA. The monolayer was scratched with a 1-ml pipette tip, and the cells were washed with PBS and cultured in 5\% FBS-DMEM. A Leica inverted phase contrast microscope (Leica DFC 300 FX; Leica Microsystems, Inc.) was used to capture images of the cells under a 20x objective lens at 0 and $24 \mathrm{~h}$. The migration of cells was determined according to the size of the wounded region.

Transwell migration/invasion assay. Cells transfected with control siRNA or TAB3 siRNA were starved overnight in
DMEM with $0.1 \%$ FBS. Subsequently, the cells were trypsinized and resuspended in DMEM containing $0.1 \%$ bovine serum albumin (Gibco; Thermo Fisher Scientific, Inc.). Cells $\left(1 \times 10^{5}\right)$ were added to the upper chambers of 24-well Transwell plates ( $8 \mu \mathrm{m}$ pore size; Corning Incorporation, Corning, NY, USA), which were used to conduct migration and invasion assays. The upper chambers of 24-well Transwell plates were not precoated with Matrigel for the migration assays; however, to observe invasive ability, the wells of the Transwell chamber were coated with Matrigel. DMEM supplemented with $10 \%$ FBS was added to the lower chambers. After overnight incubation at $37^{\circ} \mathrm{C}$, the cells that remained in the upper chamber were removed, whereas cells that had migrated/invaded to the lower chamber were fixed with $10 \%$ methanol for $20 \mathrm{~min}$ at $37^{\circ} \mathrm{C}$ and stained with $0.1 \%$ crystal violet for $30 \mathrm{~min}$ at $37^{\circ} \mathrm{C}$, in order to visualize their nuclei. The number of migrated/invaded cells in five fields was counted under 200x magnification (Leica DFC 300 FX; Leica Microsystems, Inc.), and the mean number of cells per field was determined for each chamber. All aforementioned experiments were conducted in triplicate and repeated three times.

Co-immunoprecipitation assay. TE-1 cells were harvested and lysed in buffer $(50 \mathrm{mmol} / \mathrm{l}$ Tris- $\mathrm{HCl}, \mathrm{pH} 7.5 ; 150 \mathrm{mmol} / \mathrm{l}$ $\mathrm{NaCl} ; 5 \mathrm{mmol} / \mathrm{l}$ EDTA; $1 \% \mathrm{NP}-40,0.5 \%$ deoxycholate; $0.1 \%$ SDS). A total of $30 \mu \mathrm{l}$ supernatant was collected as input. The remaining liquid was precleared with $30 \mu \mathrm{l}$ protein $\mathrm{A} / \mathrm{G}$ agarose (Santa Cruz Biotechnology, Inc.) on a rocker at $4^{\circ} \mathrm{C}$ for $2 \mathrm{~h}$. Subsequently, the precleared supernatant was separated into two samples and incubated with $6 \mu 1$ mouse monoclonal TAB3 antibody or TAK1 antibody (Santa Cruz Biotechnology, Inc.) or $0.3 \mu \mathrm{l}$ mouse immunoglobulin G (cat. no. ESK7005-96T; Sangon Biotech Co., Ltd., Shanghai, China) at $4^{\circ} \mathrm{C}$ overnight with gentle agitation. The samples were then incubated with $30 \mu \mathrm{l}$ protein $\mathrm{A} / \mathrm{G}$ at $4^{\circ} \mathrm{C}$ for $2 \mathrm{~h}$ with gentle agitation. Finally, the precipitates were collected. The immune complexes were analyzed by immunoblotting using specific antibodies against TAB3 and TAK1.

Statistical analysis. All values are expressed as the means \pm standard error of the mean. Each experiment consisted of at least three replicates per condition. Statistical analysis was performed using SPSS software (version 19.0; IBM Corp., Armonk, NY, USA). TAB3 expression and clinicopathological features were analyzed by Pearson's $\chi^{2}$ test. Spearman's correlation test was performed to analyze the correlation between TAB3 and Ki-67 expression. For analysis of survival data, Kaplan-Meier curves were constructed and log-rank tests were performed. Comparisons between two groups were conducted using Student's t-test, whereas comparisons between more than two groups were conducted using analyses of variance; one-way analysis of variance followed by Tukey's post hoc test or two-way analysis of variance followed by Bonferroni's post hoc test were conducted. $\mathrm{P}<0.05$ was considered to indicate a statistically significant difference.

\section{Results}

TAB3 is overexpressed in ESCC tissues. It has been reported that TAB3 is highly expressed in various tumors, such as 

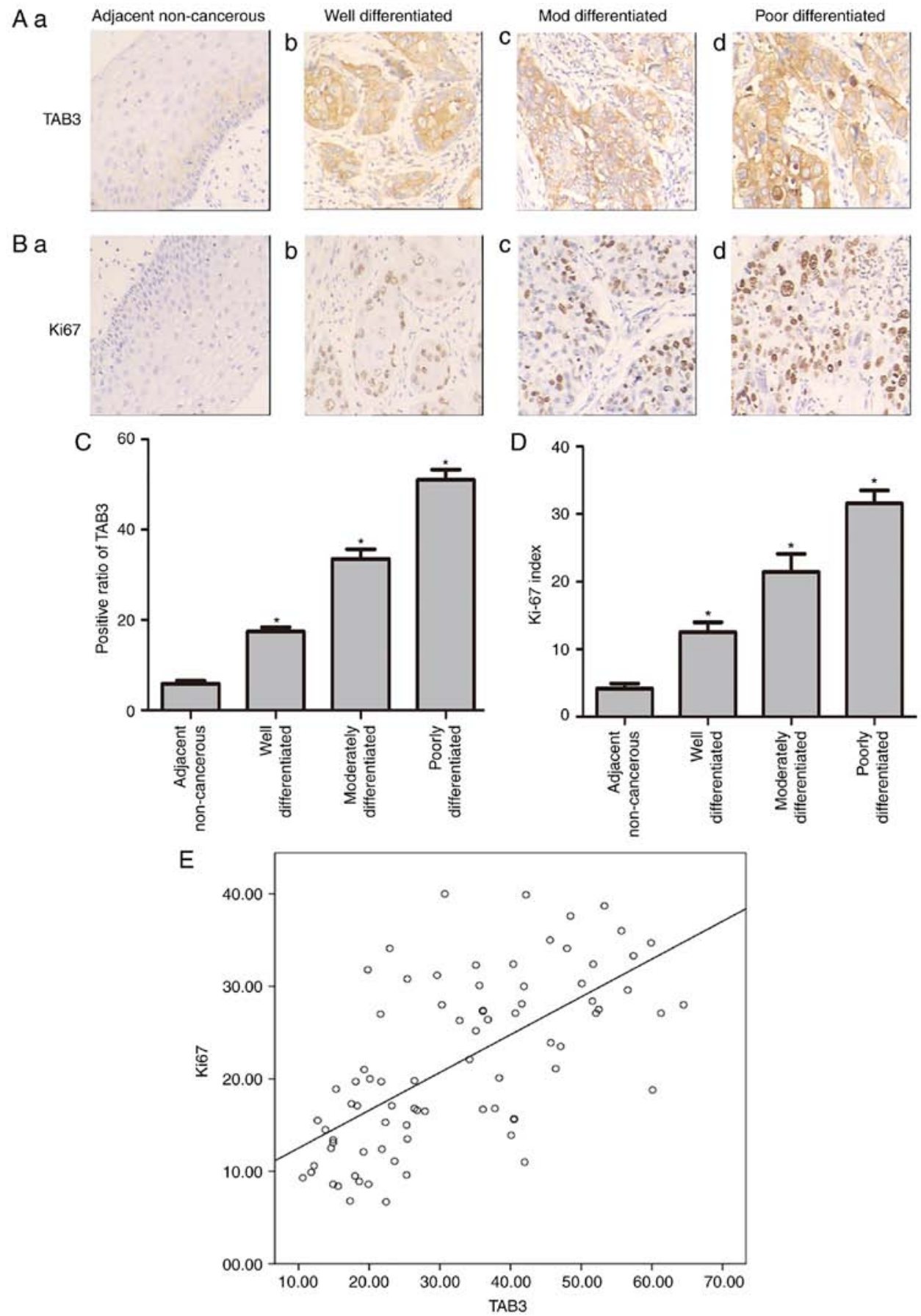

Figure 1. Overexpression of TAB3 in ESCC tissues. (A and B) TAB3 expression was examined in 80 ESCC tissues by immunohistochemical staining. Paraffin-embedded tissue sections were stained with antibodies against TAB3 and Ki-67, and were counterstained with hematoxylin. TAB3 and Ki-67 were highly expressed in ESCC. Samples were divided into (Aa and Ba) adjacent non-cancerous tissue, (Ab and Bb) well-differentiated, (Ac and Bc) moderately differentiated and (Ad and Bd) poorly differentiated groups (x200 magnification). (C) Ratio of positive TAB3 staining in adjacent non-cancerous tissue and ESCC tissues. "P<0.05 compared with adjacent non-cancerous tissues. (D) Ki-67 index in adjacent non-cancerous tissue and ESCC tissues. "P<0.05 compared with adjacent non-cancerous tissues. (E) Correlation between TAB3 and Ki-67 expression in patients with ESCC. The correlation between TAB3 and Ki-67 in ESCC was further evaluated by Spearman's rank correlation test (Spearman's $\gamma^{2}=0.419 ; \mathrm{P}=0.001$ ). ESCC, esophageal squamous cell carcinoma; TAB3, transforming growth factor-activated kinase-binding protein 3 .

non-small cell lung cancer (NSCLC), and breast and ovarian cancer (11-13). However, to the best of our knowledge, its expression has not been reported in ESCC. Therefore, immunohistochemical staining of 80 ESCC samples was performed to determine the expression of TAB3 in ESCC tissues, and to confirm the association of TAB3 with ESCC progression. As expected, TAB3 was significantly overexpressed in ESCC tissues compared with the adjacent non-cancerous tissues in most cases, and the immunoreactivity of $\mathrm{TAB} 3$ was predominantly detected in the cytoplasm (Fig. 1A). Furthermore, the positive ratio of TAB3 was upregulated in poorly differentiated ESCC tissues compared with well-differentiated tissues, which was consistent with the Ki-67 index (Fig. 1B-D). As shown in Fig. 1B and D, the Ki-67 index was significantly elevated in poorly differentiated ESCC tissues; the mean index increased from $5.1 \pm 2.2 \%$ in adjacent non-cancerous 
Table I. Expression of TAB3 in 80 human esophageal squamous cell carcinoma tissues.

TAB3

\begin{tabular}{|c|c|c|c|c|}
\hline \multirow{2}{*}{ Clinicopathological parameters } & \multirow[b]{2}{*}{ Total } & & \multirow[b]{2}{*}{ P-value } \\
\hline & & Low $(n=42)$ & High $(n=38)$ & \\
\hline \multicolumn{5}{|l|}{ Sex } \\
\hline Male & 65 & 35 & 30 & 0.616 \\
\hline Female & 15 & 7 & 8 & \\
\hline \multicolumn{5}{|l|}{ Age (years) } \\
\hline$<60$ & 26 & 17 & 9 & 0.109 \\
\hline$\geq 60$ & 54 & 25 & 29 & \\
\hline \multicolumn{5}{|l|}{ Lymph node metastasis } \\
\hline No & 46 & 31 & 15 & $0.002^{\mathrm{a}}$ \\
\hline Yes & 34 & 11 & 23 & \\
\hline \multicolumn{5}{|l|}{ T stage } \\
\hline $\mathrm{T} 1$ & 9 & 11 & 2 & $0.019^{\mathrm{a}}$ \\
\hline $\mathrm{T} 2$ & 17 & 13 & 10 & \\
\hline $\mathrm{T} 3$ & 64 & 18 & 26 & \\
\hline \multicolumn{5}{|l|}{ Pathological grade } \\
\hline Well & 25 & 15 & 10 & $0.001^{\mathrm{a}}$ \\
\hline Moderate & 34 & 23 & 11 & \\
\hline Poor & 21 & 4 & 17 & \\
\hline \multicolumn{5}{|l|}{ Ki-67 } \\
\hline Low & 32 & 22 & 10 & $0.017^{\mathrm{a}}$ \\
\hline High & 48 & 20 & 28 & \\
\hline
\end{tabular}

${ }^{a} \mathrm{P}<0.05$. Statistical analyses were performed using the Pearson's $\chi^{2}$ test. TAB3, transforming growth factor-activated kinase-binding protein 3 .

tissues to $13.2 \pm 4.3 \%$ in well-differentiated tissues, $22.1 \pm 7.2 \%$ in moderately differentiated tissues and $31.4 \pm 5.7 \%$ in poorly differentiated tissues. Subsequently, the correlation between TAB3 and Ki-67 expression was analyzed. A moderate positive correlation was determined between the expression status of TAB3 and that of Ki-67. Spearman's correlation coefficient $\left(\gamma^{2}\right)$ for TAB3-Ki-67 was $0.419(\mathrm{P}=0.001$; Fig. 1E). To further demonstrate the association between TAB3 expression and clinicopathological parameters in ESCC, the data were summarized in Table I. The expression of TAB3 was associated with lymph node metastasis $(\mathrm{P}=0.002)$, T stage $(\mathrm{P}=0.019)$, pathological grade $(\mathrm{P}=0.001)$ and Ki-67 expression $(\mathrm{P}=0.017)$, whereas there was no association with other prognostic factors.

TAB3 is overexpressed in ESCC cell lines. As shown in Fig. 1, TAB3 may be significantly overexpressed in ESCC cancerous tissues compared with in adjacent non-cancerous tissues in most cases. To determine the possible role of TAB3, western blot analysis was used to examine the protein expression levels of TAB3 HEECs and three human ESCC cell lines (TE-1, TE-10 and Eca-109). As expected, the expression levels of TAB3 were significantly higher in TE-1, TE-10 and Eca-109 cells compared with in HEEC cells (Fig. 2A and B). In addition, TE-1 cells exhibited the highest protein levels among the three ESCC cell lines; therefore, the TE-1 cell line was selected to conduct subsequent experiments.
A
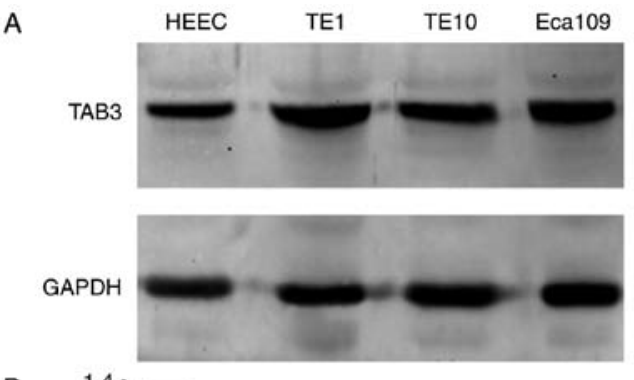

B

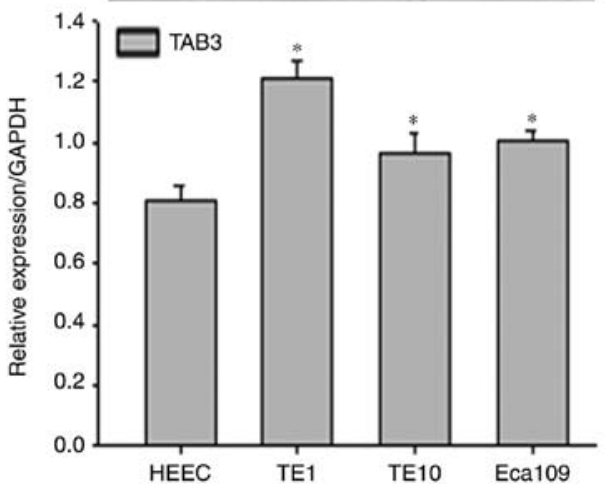

Figure 2. Overexpression of TAB3 in ESCC cell lines. (A) Protein expression levels of TAB3 were detected in HEECs and three human ESCC cell lines (TE-1, TE-10 and Eca-109) by western blotting. GAPDH was used as a loading control. (B) Relative protein expression levels of TAB3 in ESCC cells were assessed by densitometry. ${ }^{*} \mathrm{P}<0.05$ compared with HEECs. The same experiment was repeated at least three times. ESCC, esophageal squamous cell carcinoma; HEEC, human esophageal epithelial cell; TAB3, transforming growth factor-activated kinase-binding protein 3 . 


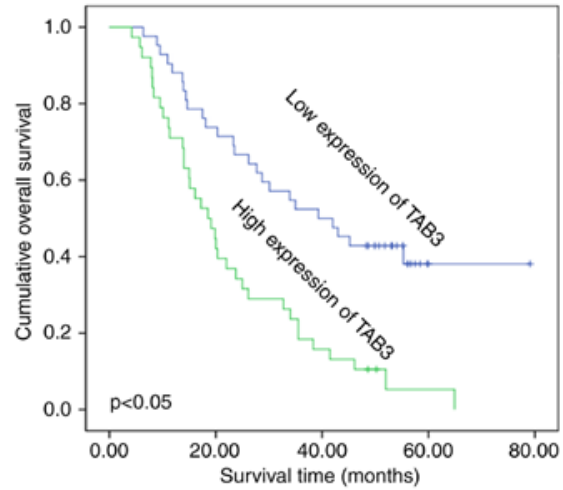

Figure 3. Association between TAB3 expression and overall survival in patients with esophageal squamous cell carcinoma. Kaplan-Meier survival analysis of TAB3 expression status. Cumulative overall survival differences between patients with high and low protein expression levels of TAB3. Patients with high TAB3 expression $(n=38)$ exhibited worse overall survival than patients with low expression $(n=42)$. TAB3, transforming growth factor-activated kinase-binding protein 3 .
Association between TAB3 expression and ESCC patient survival. The present study analyzed the association between TAB3 expression and the survival status of 80 patients using Kaplan-Meier survival curves. As shown in Fig. 3, high TAB3 expression $(n=38)$ was more significantly associated with poor survival compared with low TAB3 expression $(n=42)$. These results clearly indicated that TAB3 was associated with poor survival of patients.

Knockdown of TAB3 inhibits the proliferation of ESCC cells. To further ascertain the role of TAB3 in ESCC cell proliferation and invasion, TE-1 cells were transiently transfected with TAB3 siRNA or control siRNA for $36 \mathrm{~h}$. Western blotting was used to confirm transfection efficiency. The results of western blotting indicated that TAB3 silencing by TAB3 siRNA significantly reduced the expression levels of TAB3 in TE-1 cells compared with the control siRNA-transfected cells (Fig. 4A and B).
A

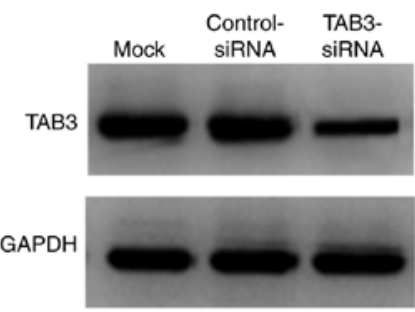

C

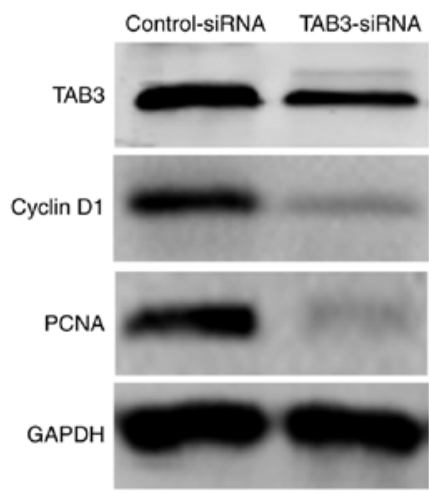

E

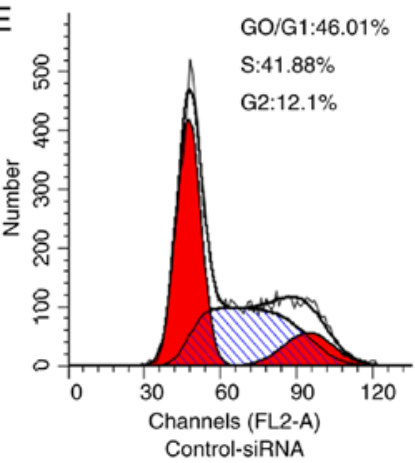

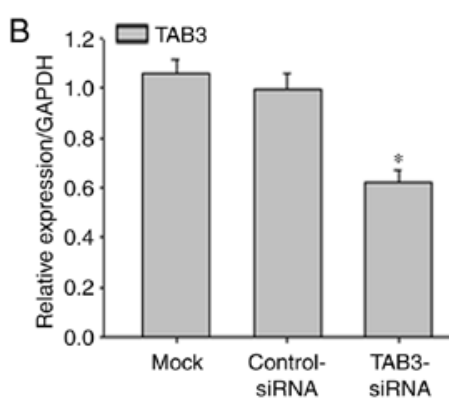

D

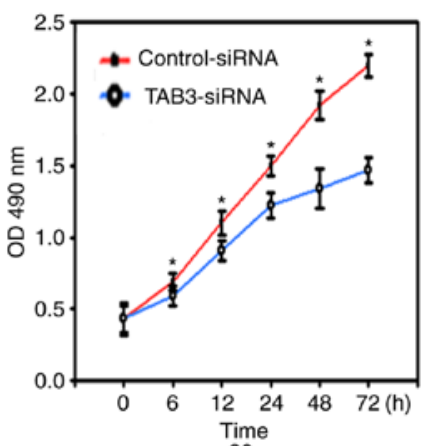

GO/G1:66.64\%

$\mathrm{S}: 14.55 \%$

G2: $18.81 \%$

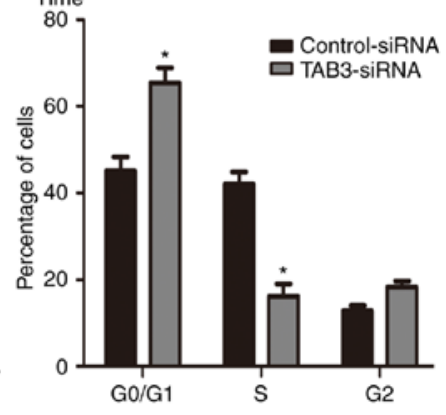

Figure 4. Knockdown of TAB3 inhibits the proliferation of ESCC cells. (A) Western blotting was performed to detect TAB3 expression levels in ESCC cells post-transfection with control siRNA or TAB3 siRNA, which resulted in the differential expression of TAB3. GAPDH was used as a loading control. (B) Protein expression levels of TAB3 were assessed by densitometry. ${ }^{*} \mathrm{P}<0.05$ compared with control siRNA group. (C) Western blotting was used to detect the expression of the cell cycle-associated proteins cyclin D1 and PCNA. The expression levels of cyclin D1 and PCNA were decreased in the TAB3 siRNA-transfected cells. GAPDH was used as a loading control. (D) ESCC cell proliferation was determined using a Cell Counting Kit-8 assay. TE-1 cells transfected with TAB3 siRNA demonstrated significantly weakened proliferation in a time-dependent manner. ${ }^{*} \mathrm{P}<0.05$ compared with the control-siRNA group. (E) Cell cycle analysis was performed following knockdown of TAB3 by TAB3 siRNA in TE-1 cells. The number of cells in $\mathrm{G}_{0} / \mathrm{G}_{1}$ phase was increased, and a concomitant reduction was observed in the number of cells in $\mathrm{S}$ phase, as determined by flow cytometry. " $\mathrm{P}<0.05$ compared with the control-siRNA group. The results are from three independent experiments. ESCC, esophageal squamous cell carcinoma; OD, optical density; PCNA, proliferating cell nuclear antigen; siRNA, small interfering RNA; TAB3, transforming growth factor-activated kinase-binding protein 3. 


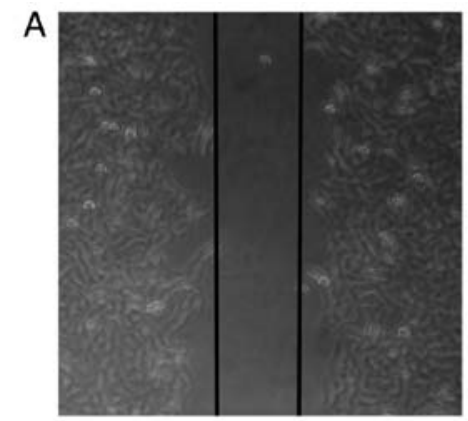

Control-siRNA

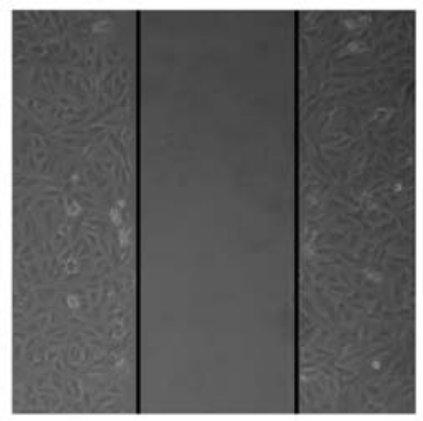

TAB3-siRNA

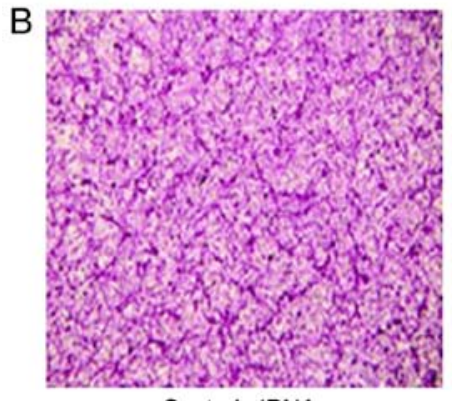

Control-siRNA

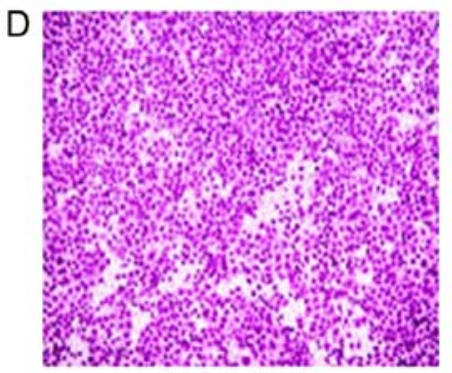

Control-siRNA
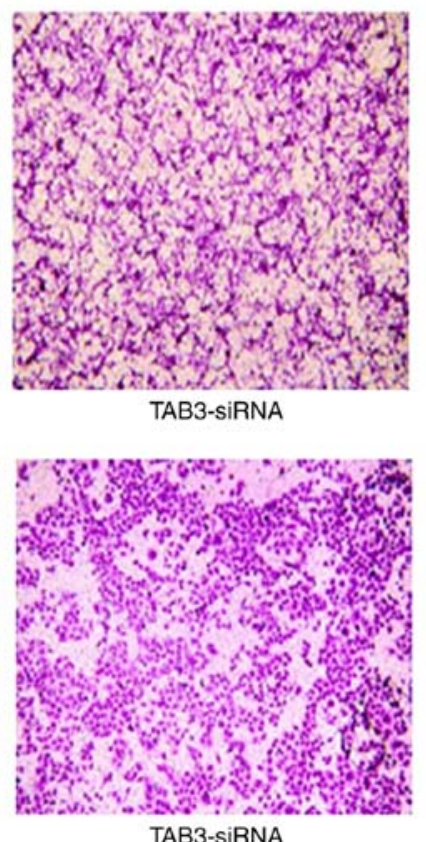
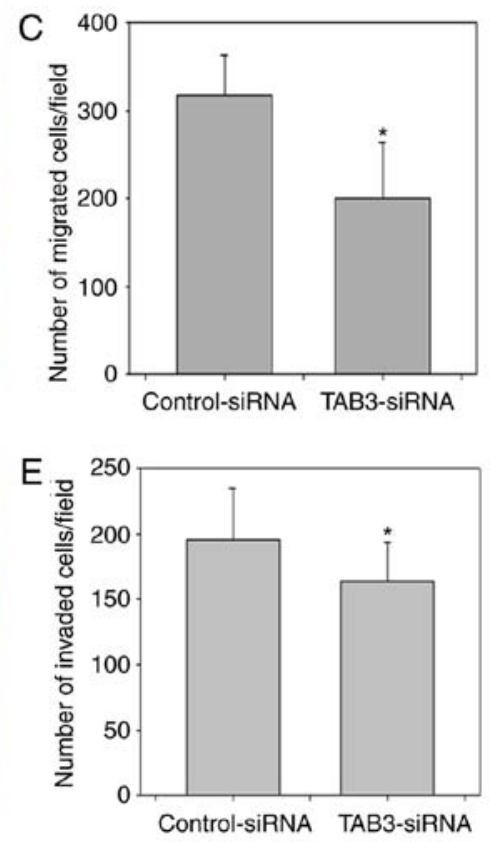

Figure 5. Knockdown of TAB3 inhibits the migration and invasion of ESCC cells. ESCC cells were transfected with control siRNA or TAB3 siRNA for $24 \mathrm{~h}$. (A) Wound healing assays were used to analyze cell migration ability. Migration of cells into the wound was visualized at $24 \mathrm{~h}$ using a Leica inverted phase contrast microscope (x200 magnification). (B and C) Cell migration was examined using a Transwell assay. Magnification, x200. (D and E) Cell invasion was examined in a Transwell chamber precoated with Matrigel. The number of cells was counted in 10 fields. Magnification, $\mathrm{x} 200$. $\mathrm{P}<0.05$ compared with the control-siRNA group. ESCC, esophageal squamous cell carcinoma; siRNA, small interfering RNA; TAB3, transforming growth factor-activated kinase-binding protein 3 .

The effects of TAB3 on cell growth were subsequently investigated. Western blot analysis demonstrated that following TAB3 downregulation via siRNA transfection, the expression levels of the cell proliferation marker PCNA and the cell cycle protein cyclin D1 were concomitantly inhibited (Fig. 4C). In addition, the CCK-8 assay demonstrated that knockdown of TAB3 significantly reduced cell proliferation in a time-dependent manner (Fig. 4D). Furthermore, flow cytometric analysis was conducted to reveal cell cycle distribution. The percentage of TE- 1 cells arrested in $\mathrm{G}_{0} / \mathrm{G}_{1}$ phase increased to $66.64 \%$ post-transfection with TAB3 siRNA, whereas the percentage of cells in S phase decreased from 41.88 to $14.55 \%$, thus suggesting that TAB3 may be able to promote the $\mathrm{G}_{0} / \mathrm{G}_{1}-\mathrm{S}$ transition and thus promote cell growth (Fig. 4E). These results suggested that the expression of TAB3 may promote ESCC cell proliferation.

Knockdown of TAB3 reduces the migration and invasion of ESCC cells. Cancer cell motility is known to be indispensable for cancer metastasis and invasion. Therefore, the present study aimed to determine the effects of TAB3 on the migration and invasion of ESCC cells. Wound healing and Transwell assays were performed to assess the effects of TAB3 on cell migration and invasion. As shown in Fig. 5A, knockdown of TAB3 markedly decreased the rate of the wound healing process. Subsequently, the Transwell migration assay was used to confirm that TAB3 knockdown inhibited the migration of TE-1 cells (Fig. 5B and C). The results suggested that knockdown of TAB3 reduced the migration of TE-1 cells. Finally, Transwell invasion assays were conducted to determine whether knockdown of TAB3 reduced the invasion of ESCC cells. As shown in Fig. 5D and E, knockdown of TAB3 significantly reduced the number of TE- 1 cells invading through the Matrigel-coated polycarbonate filter to the lower chamber. These results indicated that TAB3 may increase the migration and invasion of TE- 1 cells.

Knockdown of TAB3 decreases the expression of the NF- $\kappa B$ pathway in TE-1 cells. It has been reported that TAB3 binds to TAK1; the compound is then phosphorylated, leading 
A

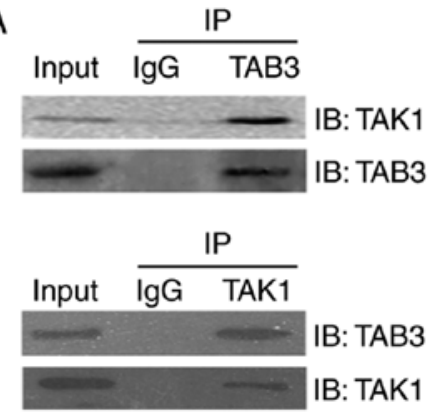

B

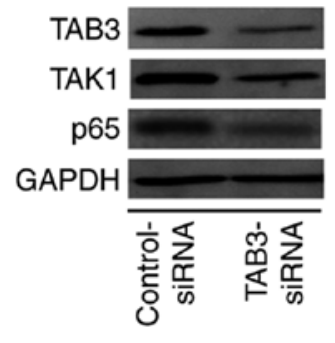

Figure 6. Knockdown of TAB3 decreases the expression of the nuclear factor- $\kappa$ B pathway in TE-1 cells. (A) Interaction between TAB3 and TAK1 in TE-1 cells was demonstrated by a co-IP assay. The immune complexes were analyzed by western blotting with the indicated antibodies. (B) TE-1 cells were transfected with control siRNA or TAB3 siRNA, and western blotting was used to analyze the protein expression levels of TAB3 TAK1 and p65. The same experiment was repeated at least three times. IB, immunoblotting IgG, immunoglobulin G; IP, immunoprecipitation; siRNA, small interfering RNA; TAB3, TAK1-binding protein 3; TAK1, transforming growth factor-activated kinase.

to activation of the NF- $\mathrm{B}$ pathway $(15,16)$. The present study analyzed the TAB3-TAK1-NF- $\mathrm{B}$ pathway in ESCC cells. To further study the mechanism of TAB3 in ESCC, co-immunoprecipitation was performed to determine the relationship between TAB3 and TAK1; the results revealed that TAB3 interacted with TAK1 in TE-1 cells (Fig. 6A). Subsequently, western blotting was used to determine whether TAB3-TAK1 compounds could influence the NF- $\mathrm{BB}$ pathway. The results demonstrated that silencing TAB3 with TAB3 siRNA decreased the expression of p65, which is a member of the NF- $\kappa$ B family (Fig. 6B). These results suggested that TAB3 may interact with TAK1 and subsequently upregulate the $N F-\kappa B$ pathway.

\section{Discussion}

The present study demonstrated that TAB3 was significantly upregulated in human ESCC tissues and cell lines, and that TAB3 expression was associated with poor survival of patients. In addition, knockdown of TAB3 expression by RNA interference inhibited the proliferation and invasion of ESCC cells. Finally, TAB3 was revealed to affect ESCC through activation of the TAK1-NF- $\kappa \mathrm{B}$ pathway. Therefore, TAB3 may be a promising therapeutic target for the treatment of ESCC.

It has been reported that TAB3 is overexpressed in several types of cancer, and that it is associated with tumor development and invasion. Tao et al (12) used western blot analysis to demonstrate that p38 mitogen-activated protein kinase (MAPK) induces TAB3 and enhances TAB3-mediated $\mathrm{NF}-\kappa \mathrm{B}$ activation in breast cancer. Furthermore, it was revealed that the expression of TAB3 increases cell migration and invasion by activating $\mathrm{NF}-\kappa \mathrm{B}$, and TAB3 expression is significantly correlated with poor outcomes of patients (12). Another study demonstrated that in ovarian cancer, TAB3 is significantly overexpressed, enhancing cancer cell proliferation and reducing chemical sensitivity (13). Our previous study revealed that in NSCLC, TAB3 overexpression promotes

cell proliferation and mediates chemoresistance to cisplatin via the NF- $\mathrm{NB}$ pathway (11). TAB3 is involved in several types of tumor; however, to the best of our knowledge, its effect on ESCC remains to be studied. Therefore, the present study is the first to observe that the expression of TAB3 may be markedly upregulated in ESCC cells, thus suggesting that TAB3 may serve a role in the development and progression of ESCC.

The cell cycle is associated with cell transformation and carcinogenesis (17-19). Boonstra and Moes demonstrated that the MAPK and phosphoinositide 3-kinase pathways have an essential role in early $G_{1}$ phase (18), and cyclin D1 is a key target of proliferative signals in $G_{1}$ phase (20). The present study demonstrated that following knockdown of TAB3 with siRNA, the expression levels of PCNA and cyclin D1 were concomitantly inhibited; therefore, TAB3 may be involved in carcinogenesis and the development of ESCC. To the best of our knowledge, the effects of TAB3 on other cell cycle control molecules, such as cyclin-dependent kinase (cdk)4, cdk6, p21CIP1, p27KIP1 and p53, have not been reported. The present study did not analyze these molecules; therefore, further studies may elucidate the effect of TAB3 on more cell cycle control molecules.

Increasing evidence has revealed that the $\mathrm{NF}-\kappa \mathrm{B}$ pathway serves a key role in promoting ESCC progression by modulating cell cycle and invasion processes (21-24). Activation of the $\mathrm{NF}-\kappa \mathrm{B}$ pathway induces epithelial-mesenchymal transition in cells $(25,26)$. Furthermore, it has been reported that activation of both the activator protein 1 (AP-1) and the NF- $\kappa \mathrm{B}$ transcription factors may increase the invasive properties of ESCC cells $(27,28)$.

TAB3 was initially identified as the human ortholog of Xenopus TAB3 in the NF- $\mathrm{BB}$ pathway and as a hypothetical oncogene (29). Both AP-1 and NF- $\kappa$ B transcription factors can be activated by overexpression of TAB3 (29). Numerous studies have implicated TAB3 as a central factor in cell survival, proliferation and metastasis, which are actions that are mediated by activating the $\mathrm{NF}-\kappa \mathrm{B}$ pathway $(11,12,16)$. TAB3 and its homolog TAK1-binding protein 2 (TAB2) are adaptor molecules linking tumor necrosis factor (TNF)-associated factor 6 , tumor necrosis factor $\alpha$ and interleukin-1, which are TNF-associated factor molecules. Both TAB2 and TAB3 can activate TAK1. Previous reports have demonstrated that inhibition of TAK1 may be an effective target for the induction of cancer cell death $(9,29)$. The present study also revealed that knockdown of TAB3 significantly inhibited proliferation and invasion of ESCC cells via the $\mathrm{NF}-\kappa \mathrm{B}$ pathway. Based on these findings, it may be concluded that TAB3 participates in $\mathrm{NF}-\kappa \mathrm{B}$ pathway signaling in ESCC. However, another way to determine whether TAB3 activates the $\mathrm{NF}-\kappa \mathrm{B}$ pathway is to examine phosphorylated-p65 expression using nuclear extracts. As a limitation of the present study, our future studies aim to examine phosphorylated-p65.

In conclusion, the present study demonstrated that high TAB3 expression was markedly associated with poor survival. Furthermore, knockdown of TAB3 expression inhibited the proliferation and invasion of ESCC cells by suppressing the $\mathrm{NF}-\kappa \mathrm{B}$ pathway, which may represent a novel therapeutic target for the treatment of ESCC. However, it has been 
suggested that knockdown of TAB3 leads to chemoresistance in ovarian cancer and NSCLC $(11,13)$. Radiotherapy and chemotherapy are important therapeutic strategies in the treatment of ESCC. Therefore, future studies, potentially using TAB3 knockout mice to investigate the function of TAB3 in tumor radiotherapy and chemotherapy in vivo, may further elucidate the functional significance of this gene in the NF- $\mathrm{B}$ signaling pathway.

\section{Acknowledgements}

The authors would like to thank Dr Jian Zhao and Dr Dan Dan for their valuable input.

\section{Funding}

The present study was supported by the National Natural Science Foundation of China (grant nos. 31670857 and 31700737), the Natural Science Foundation of Jiangsu Province (grant no. BK20161152), the Key Scientific Research Program of Wuxi Municipal Health Bureau (grant no. Z201509), the Program for Innovative Research of Wuxi (grant no. CXTD004), and the Young Talents Subsidy Project of Wuxi Municipal Commission of Health and Family Planning (grant no. QNRC092).

\section{Availability of data and materials}

The datasets used and/or analyzed during the current study are available from the corresponding author on reasonable request.

\section{Authors' contributions}

JZ performed the histological examination of the tissues and was a major contributor in writing the manuscript. LG performed the wound healing assay and Transwell migration/invasion assays. YG performed the western blotting and co-immunoprecipitation assay. WX performed the cell proliferation assays. DS, QL and WM analyzed the possible mechanism by which TAB3 participated in tumorigenesis of ESCC. FW performed the cell cycle analysis. PL and JC designed the present study. All authors read and approved the final manuscript.

\section{Ethics approval and consent to participate}

All patients provided written informed consent for their tissue samples to be used for scientific research. The present study was approved by the Affiliated Hospital of Nantong University Ethics Committee (Nantong, China).

\section{Patient consent for publication}

All patients that participated in this study provided informed consent for publication.

\section{Competing interests}

The authors declare that they have no competing interests.

\section{References}

1. Chen W, Zheng R, Baade PD, Zhang S, Zeng H, Bray F, Jemal A, $\mathrm{Yu}$ XQ and He J: Cancer statistics in China, 2015. CA Cancer J Clin 66: 115-132, 2016.

2. Siegel RL, Miller KD and Jemal A: Cancer statistics, 2017. CA Cancer J Clin 67: 7-30, 2017.

3. Yano T, Muto M, Minashi K, Iwasaki J, Kojima T, Fuse N, Doi T, Kaneko K and Ohtsu A: Photodynamic therapy as salvage treatment for local failure after chemoradiotherapy in patients with esophageal squamous cell carcinoma: A phase II study. Int J Cancer 131: 1228-1234, 2012.

4. Zhang L, Wu YD, Li P, Tu J, Niu YL, Xu CM and Zhang ST: Effects of cyclooxygenase-2 on human esophageal squamous cell carcinoma. World J Gastroenterol 17: 4572-4580, 2011.

5. Lagergren J, Smyth E, Cunningham D and Lagergren P: Oesophageal cancer. Lancet 390: 2383-2396, 2017.

6. Rustgi AK and El-Serag HB: Esophageal carcinoma. N Engl J Med 371: 2499-2509, 2014.

7. Enzinger PC and Mayer RJ: Esophageal cancer. N Engl J Med 349: 2241-2252, 2003.

8. Takaesu G, Kishida S, Hiyama A, Yamaguchi K, Shibuya H, Irie $\mathrm{K}$, Ninomiya-Tsuji $\mathrm{J}$ and Matsumoto K: TAB2, a novel adaptor protein, mediates activation of TAK1 MAPKKK by linking TAK1 to TRAF6 in the IL-1 signal transduction pathway. Mol Cell 5: 649-658, 2000.

9. Besse A, Lamothe B, Campos AD, Webster WK, Maddineni U, Lin SC, Wu H and Darnay BG: TAK1-dependent signaling requires functional interaction with $\mathrm{TAB} 2 / \mathrm{TAB} 3$. J Biol Chem 282: 3918-3928, 2007.

10. Hu Y, Liu JP, Zhu Y and Lu NH: The importance of toll-like receptors in NF- $\kappa \mathrm{B}$ signaling pathway activation by helicobacter pylori infection and the regulators of this response. Helicobacter 21: 428-440, 2016.

11. Chen J, Gu J, Feng J, Liu Y, Xue Q, Ni T, Wang Z, Jia L, Mao G and Ji L: TAB3 overexpression promotes cell proliferation in non-small cell lung cancer and mediates chemoresistance to CDDP in A549 cells via the NF- $\mathrm{kB}$ pathway. Tumour Biol 37: 3851-3861, 2016.

12. Tao T, He Z, Shao Z and Lu H: TAB3 O-GlcNAcylation promotes metastasis of triple negative breast cancer. Oncotarget 7 : 22807-22818, 2016.

13. Chen Y, Wang X, Duan C, Chen J, Su M, Jin Y, Deng Y, Wang D, Chen C, Zhou L, et al: Loss of TAB3 expression by shRNA exhibits suppressive bioactivity and increased chemical sensitivity of ovarian cancer cell lines via the NF- $\kappa \mathrm{B}$ pathway. Cell Prolif 49: 657-668, 2016.

14. Sobin LH, Gospodarowicz MK and Wittekind C: TNM classification of malignant tumours. John Wiley \& Sons, 2011.

15. Roh YS, Song J and Seki E: TAK1 regulates hepatic cell survival and carcinogenesis. J Gastroenterol 49: 185-194, 2014.

16. Luo C, Yuan R, Chen L, Zhou W, Shen W, Qiu Y, Shao J, Yan J and Shao J: TAB3 upregulates Survivin expression to promote colorectal cancer invasion and metastasis by binding to the TAK1-TRAF6 complex. Oncotarget 8: 106565-106576, 2017.

17. Malumbres $\mathrm{M}$ and Carnero A: Cell cycle deregulation: A common motif in cancer. Prog Cell Cycle Res 5: 5-18, 2003.

18. Boonstra $\mathbf{J}$ and Moes MJ: Signal transduction and actin in the regulation of G1-phase progression. Crit Rev Eukaryot Gene Expr 15: 255-276, 2005.

19. Hulleman E and Boonstra J: Regulation of G1 phase progression by growth factors and the extracellular matrix. Cell Mol Life Sci 58: 80-93, 2001.

20. Tashima Y, Hamada H, Okamoto $M$ and Hanai T: Prediction of key factor controlling G1/S phase in the mammalian cell cycle using system analysis. J Biosci Bioeng 106: 368-374, 2008.

21. Gan J, Ke X, Jiang J, Dong H, Yao Z, Lin Y, Lin W, Wu X, Yan S, Zhuang Y, et al: Growth hormone-releasing hormone receptor antagonists inhibit human gastric cancer through downregulation of PAK1-STAT3/NF-kappaB signaling. Proc Natl Acad Sci USA 113: 14745-14750, 2016

22. Jung JU, Ravi S, Lee DW, McFadden K, Kamradt ML, Toussaint LG and Sitcheran R: NIK/MAP3K14 regulates mitochondrial dynamics and trafficking to promote cell invasion. Curr Biol 26: 3288-3302, 2016.

23. Gan X, Chen B, Shen Z, Liu Y, Li H, Xie X, Xu X, Li H, Huang Z and Chen J: High GPX1 expression promotes esophageal squamous cell carcinoma invasion, migration, proliferation and cisplatin-resistance but can be reduced by vitamin D. Int J Clin Exp Med 7: 2530-2540, 2014 
24. Han Y, Guo XH, Zheng QF, Zhu YL, Fan YY and Zhang XY: Down-regulation of platelet-derived growth factor-D expression blockades NF- $\kappa \mathrm{B}$ pathway to inhibit cell proliferation and invasion as well as induce apoptosis in esophageal squamous cell carcinoma. Mol Biol Rep 40: 2473-2483, 2013.

25. Gao S, Sun Y, Zhang X, Hu L, Liu Y, Chua CY, Phillips LM, Ren H, Fleming JB, Wang H, et al: IGFBP2 activates the NF- $\kappa$ B pathway to drive epithelial-mesenchymal transition and invasive character in pancreatic ductal adenocarcinoma. Cancer Res 76: 6543-6554, 2016.

26. Pires BR, Mencalha AL, Ferreira GM, de Souza WF, Morgado-Díaz JA, Maia AM, Corrêa S and Abdelhay ES NF-kappaB is involved in the regulation of EMT genes in breast cancer cells. PLoS One 12: e0169622, 2017.
27. Shin WS, Hong Y, Lee HW and Lee ST: Catalytically defective receptor protein tyrosine kinase PTK7 enhances invasive phenotype by inducing MMP-9 through activation of AP-1 and NF- $\kappa \mathrm{B}$ in esophageal squamous cell carcinoma cells. Oncotarget 7: 73242-73256, 2016.

28. Zhou J, Zheng S, Liu T, Liu Q, Chen Y, Tan D, Ma R and Lu X: $\mathrm{MCP} 2$ activates $\mathrm{NF}-\kappa \mathrm{B}$ signaling pathway promoting the migration and invasion of ESCC cells. Cell Biol Int 42: 365-372, 2018.

29. Jin G, Klika A, Callahan M, Faga B, Danzig J, Jiang Z, Li X, Stark GR, Harrington J and Sherf B: Identification of a human NF-kappaB-activating protein, TAB3. Proc Natl Acad Sci USA 101: 2028-2033, 2004. 